Revue musicale OICRM

revue musicale oicrm

\title{
La création d'Another Brick in the Wall - l'opéra de Julien Bilodeau. Les différents enjeux du crossover entre opéra et rock
}

\section{Danick Trottier}

Volume 4, numéro 2, 2017

URI : https://id.erudit.org/iderudit/1043224ar

DOI : https://doi.org/10.7202/1043224ar

Aller au sommaire du numéro

Éditeur(s)

Observatoire interdisciplinaire de création et recherche en musique (OICRM)

ISSN

2368-7061 (numérique)

Découvrir la revue

Citer cet article

Trottier, D. (2017). La création d'Another Brick in the Wall - l'opéra de Julien Bilodeau. Les différents enjeux du crossover entre opéra et rock. Revue musicale OICRM, 4(2), 136-161. https://doi.org/10.7202/1043224ar
Résumé de l'article

En date du 11 mars 2017 avait lieu à Montréal la création d'Another Brick in the Wall- l'opéra du compositeur Julien Bilodeau. Représentée par l'Opéra de Montréal dans le contexte du $375^{\mathrm{e}}$ anniversaire de fondation de Montréal, l'oeuvre a été l'objet d'une importante campagne de publicité et a mobilisé l'attention des médias, comme l'a rarement fait une création opératique dans l'histoire de Montréal. Cette attention est aussi secondée par le crossover classique qui est à l'origine de l'oeuvre, soit le fait de métamorphoser The Wall en opéra avec l'accord de son principal créateur Rogers Waters. L'opéra de Bilodeau est donc à l'origine d'une multitude de questions dans son rapport à l'oeuvre d'origine, ces questions se posant autant du côté de la production avec le langage musical adopté par le compositeur que du côté de la réception avec le croisement des conventions appartenant à l'opéra et au rock. L'article met en relief ces questions et tente d'y répondre en prenant en considération le phénomène du crossover entre opéra et rock, la partition de Bilodeau ainsi que les critiques publiées dans la foulée de la première. 


\title{
La création d'Another Brick in the Wall - l'opéra de Julien Bilodeau. Les différents enjeux du crossover entre opéra et rock
}

\author{
Danick Trottier
}

\begin{abstract}
Résumé
En date du 11 mars 2017 avait lieu à Montréal la création d'Another Brick in the Wall - l'opéra du compositeur Julien Bilodeau. Représentée par l'Opéra de Montréal dans le contexte du $375^{\mathrm{e}}$ anniversaire de fondation de Montréal, l'œuvre a été l'objet d'une importante campagne de publicité et a mobilisé l'attention des médias, comme l'a rarement fait une création opératique dans l'histoire de Montréal. Cette attention est aussi secondée par le crossover classique qui est à l'origine de l'œuvre, soit le fait de métamorphoser The Wall en opéra avec l'accord de son principal créateur Rogers Waters. L'opéra de Bilodeau est donc à l'origine d'une multitude de questions dans son rapport à l'œuvre d'origine, ces questions se posant autant du côté de la production avec le langage musical adopté par le compositeur que du côté de la réception avec le croisement des conventions appartenant à l'opéra et au rock. L'article met en relief ces questions et tente d'y répondre en prenant en considération le phénomène du crossover entre opéra et rock, la partition de Bilodeau ainsi que les critiques publiées dans la foulée de la première.
\end{abstract}

Mots clés : Julien Bilodeau ; crossover classique ; opéra contemporain ; The Wall ; Roger Waters.

\begin{abstract}
On March 11, 2017, the creation of Another Brick in the Wall-The Opera by composer Julien Bilodeau took place in Montreal. Performed by the Opéra de Montréal in context of Montreal's birthday for its $375^{\text {th }}$ anniversary, the work was deployed through an amazing campaign of advertising in addition to the attention given by the media, as it was never been the case before for a creation of that kind in Montreal. That attention was also the result of the classical crossover at the core of the work, namely the metamorphosis of The Wall into a truly opera with the agreement of its principal leader Roger Waters. The opera composed by Bilodeau is then at the confluence of a plethora of questions in front of The Wall, these questions being on the side of production through the composer's choices for musical language as well as on the side of reception
\end{abstract}


though the crossover of conventions from opera and rock. The article addresses these questions regarding the phenomena of classical crossover between opera and rock, Bilodeau's score and the critiques published in the aftermath of the première.

Keywords: Julien Bilodeau; classical crossover; contemporary opera; The Wall; Roger Waters.

Another Brick in the Wall -l'opéra du compositeur canadien Julien Bilodeau a été créé le 11 mars $2017^{1}$. Représentée dix fois dans le cadre de la programmation régulière de l'Opéra de Montréal, la production de deux heures a été conçue « d'après les paroles et la musique de The Wall ${ }^{2}$ ", soit le double album sorti en 1979 par Pink Floyd, mais dont la paternité est rattachée à la figure de Roger Waters. Montréal a ainsi vibré au rythme de l'opéra comme elle l'a rarement fait par le passé, et la présence de Waters comme caution morale y était pour beaucoup. C'est que l'œuvre était aussi présentée comme l'un des moments phares des festivités entourant le $375^{\mathrm{e}}$ anniversaire de la ville $^{3}$, ce pour quoi le budget alloué à cette production a largement dépassé ce qu'on retrouve normalement pour ce type de création au Québec ${ }^{4}$.

De fait, l'œuvre ne peut être uniquement rattachée à cet anniversaire puisqu'elle prolonge aussi un contexte de création qui met en valeur l'opéra depuis quelques années. C'est ainsi que la scène lyrique du Québec a été augmentée de plusieurs titres depuis l'arrivée des années 2000, par exemple L'arche (2004) d'Isabelle Panneton, L'eau qui danse, la pomme qui chante et l'oiseau qui dit la vérité (2009) de Gilles Tremblay, Le rêve de Grégoire (2014) de Pierre Michaud ainsi que Les Feluettes (2016) de Kevin March ${ }^{5}$. Cette dernière œuvre a été créée lors de la saison 2015-2016 de 1'Opéra de Montréal et témoigne ainsi de l'intérêt de l'institution pour de nouvelles créations.

La production a aussi misé sur le talent local pour vendre l'œuvre et attirer le grand public, à commencer par la conception et la mise en scène assumées par

1 J'ai rencontré Julien Bilodeau le 20 mars 2017 au Café du Nouveau Monde à Montréal. Nos échanges, que j'ai évités de formaliser sous la forme d'un questionnaire ou d'un entretien dirigé, ont porté sur l'opéra - ils ont été suivis d'échanges par courriels dans la foulée de l'écriture du présent article. C'est dans ce contexte que le compositeur m'a donné accès à la partition (Bilodeau 2017). Je tiens donc ici à le remercier pour les informations transmises et pour son soutien logistique. Les exemples musicaux reproduits dans le cadre de cet article sont le fruit de sa collaboration.

2 «D'après les paroles et la musique de The Wall de Roger Waters » est la mention qui se trouve sur la page titre de la partition de Bilodeau (2017, page-titre).

3 Le lien unissant Waters, The Wall, Montréal, et ayant justifié la création, sera expliqué en détail plus loin dans le texte.

4 Le chiffre donné par l'Opéra de Montréal et repris dans les médias est de 3,1 millions de dollars canadiens (voir Houle 2017).

5 À la lumière des travaux d'Eric Salzman et Thomas Desi (2008), il convient d'établir une distinction entre théâtre musical et opéra, même si les auteurs admettent que cette démarcation est parfois ténue. Les œuvres de Tremblay et Michaud s'inscrivent davantage dans le contexte du théâtre musical pour la place qu'elles confèrent à la dimension théâtrale, à l'effectif réduit et à la voix. À l'inverse, l'œuvre de Bilodeau discutée ici évolue davantage dans les frontières de l'opéra traditionnel. Dans leur livre, Salzman et Desi donnent un aperçu de la vitalité qu'a connue le théâtre musical au Québec depuis les années 1970 (Salzman et Desi 2008, p. 213-214). 
Dominic Champagne, ainsi que les rôles dévolus à l'Orchestre Métropolitain et au Chœur de 1'Opéra de Montréal sous la direction d'Alain Trudel - le philosophe Normand Baillargeon a aussi participé à la production comme conseiller dramaturgique, alors que le compositeur Louis Dufort a pris en charge le design sonore de l'œuvre (par exemple, les sons associés à la guerre). Les personnages qui normalement tiennent un rôle secondaire dans le double album ont été approfondis quant à leur rôle dramatique ; c'est une des facettes majeures de l'œuvre qui sera discutée plus loin. L'opéra comprend ainsi neuf personnages, incluant Pink comme protagoniste. Ces personnages ont tous été assumés par des artistes lyriques du Québec, certains étant plus en vue sur la scène internationale que d'autres, par exemple Étienne Dupuis (baryton) dans le rôle de Pink. Les autres personnages se déclinent ainsi : la mère par France Bellemare (mezzo-soprano), le père par Jean-Michel Richer (ténor), la conjointe de Pink par Caroline Bleau (soprano), Vera Lynn par Stéphanie Pothier (mezzo-soprano), le professeur par Dominic Lorange (ténor), à la fois le procureur et le médecin par Geoffroy Salvas (baryton), et le juge par Marcel Beaulieu (basse). Tous ces ingrédients réunis ont permis de créer un engouement médiatique autour de l'événement ${ }^{6}$.

Les dix représentations et l'attention accordée à cette production ne peuvent faire oublier le défi de taille auquel faisait face une telle création : comment s'émanciper de l'œuvre d'origine et parvenir à un véritable opéra ? La nouvelle œuvre de Bilodeau repose sur la trame narrative de l'album double de Pink Floyd en reprenant les 26 chansons tout en apportant des modifications importantes aux niveaux musical et dramatique, transformant ainsi l'œuvre d'origine en opéra comme il sera analysé dans les lignes à venir. Cette rencontre n'est pas fortuite puisque l'opéra et le rock partagent quelques points en commun, dont certains seront discutés plus loin. Or, le phénomène du crossover en musique, plus particulièrement le crossover classique dans ce cas-ci ${ }^{7}$, ne va pas de soi tant le chemin est parsemé d'embûches, à commencer par les négociations pour les droits d'auteurs et les choix stylistiques dans le passage d'un genre à un autre. Pourtant, les phénomènes de crossover ne datent pas d'hier, tout comme les croisements entre musique contemporaine et rock $^{8}$. Dans le cas de Bilodeau et de

6 Plusieurs recensions critiques ont été publiées dans les journaux, autant au niveau local qu'au niveau international. Par exemple, les quatre grands quotidiens montréalais que sont Le Journal de Montréal, La Presse, Montreal Gazette et Le Devoir ont publié chacun un minimum de deux critiques. Certaines de ces critiques seront commentées ou citées à témoin plus loin dans le texte.

7 La notion de crossover est utilisée dans la culture populaire pour désigner les croisements et les phénomènes d'hybridation entre genres ou styles musicaux, ce qui est le cas ici dans le passage d'une œuvre de la culture rock à la culture opératique - l'hybridation à partir du classique est nommée crossover classique. Le but de la présente étude n'est pas d'approfondir la notion de crossover, ses implications épistémiques ou le genre qu'elle circonscrit, mais bien de comprendre les rencontres possibles entre opéra et rock et la manière dont le crossover à la base de l'œuvre étudiée a été conçue. Selon Paul Fryer, les concerts hautement médiatisés offerts par les Trois Ténors (Pavarotti, Domingo et Carreras) à partir des années 1990 marquent un tournant dans le cas du crossover classique en devenant un phénomène de masse (Fryer 2014, p. 128).

8 La revue Circuit, musiques contemporaines s'est penchée sur le phénomène d'hybridation entre musiques contemporaines et musiques rock autour de la figure de Fausto Romitelli et de l'inclusion de la guitare électrique dans ses œuvres (voir Goldman 2014) - il est à noter que Romitelli a exercé une influence 
l'équipe de production, les défis de l'entreprise ont consisté dans la métamorphose du double album en opéra à part entière, autrement Waters n'aurait jamais donné son accord au projet. Car ce dernier s'est toujours opposé à des adaptations musicales de The Wall. Lorsqu'en décembre 2014, Bilodeau, Champagne et Pierre Dufour (ancien directeur de l'Opéra de Montréal qui est à l'origine du projet) sont allés à sa rencontre pour le convaincre d'embarquer dans l'aventure, la condition pour un accord était la création d'une nouvelle œuvre (voir Dufour 2017). De telle sorte que le crossover qui a donné lieu à une nouvelle œuvre (plutôt qu'une simple adaptation symphonique) est aussi redevable aux conditions posées par Waters.

Les prochaines lignes se pencheront donc sur la nature de cette métamorphose et ce qu'elle nous dit du crossover qui se joue dans le passage de The Wall à Another Brick in the Wall-l'opéra. Mais cette métamorphose ne va pas sans risque et sans difficulté, entre autres en raison de la notoriété de l'œuvre d'origine, ce qui a provoqué certains malentendus du côté de la réception. Il est donc important d'approfondir les enjeux que porte ce genre de crossover entre opéra et rock tout en cernant les difficultés qui se posent dans le passage de la production à la réception.

\section{LE CROSSOVER ENTRE OPÉRA ET ROCK}

Quelques-unes des passations qui sont à l'œuvre entre l'opéra et le rock doivent être mises en relief pour bien comprendre ce qui est en jeu dans Another Brick in the Wall - l'opéra, notamment la forme d'appropriation qu'exige la métamorphose recherchée par Bilodeau et l'équipe de production. Ainsi pourra-t-on mieux cerner les valeurs qui fondent le rock que Waters a pratiqué depuis sa jeunesse et qui, à terme, peuvent aussi expliquer les raisons pour lesquelles il a répondu positivement à cette proposition d'opéra.

L'un de ces points de conjonction entre opéra et rock est le courant de l'art rock qui va essaimer dans le contexte des années 1960, notamment dans la foulée de l'album concept ${ }^{9}$. L'art rock, comme son nom l'indique, cherche à faire du rock un art officiel en mettant en valeur l'expérimentation sonore, la virtuosité instrumentale et le sens du message derrière les paroles, d'où l'importance de la formation classique de ses musiciens et les rencontres recherchées avec la tradition classique - de fait, l'art rock est aussi nommé le classical rock (Lipscomb et Stuessy [2001]2013, p. 205-206), ce qui est l'équivalent de ce qu'on nomme en français le rock classique. C'est ainsi que les idéaux artistiques qui circonscrivent ce type de rock concourent à sa légitimité

importante sur Bilodeau, comme il sera discuté plus loin dans le texte. Un autre cas de figure qui pourrait être évoqué par rapport à cette hybridation est celui de Frank Zappa, dont l'inclusion dans l'univers des musiques dites savantes a été une réalité indéniable au cours des dernières décennies (voir Beaucage 2004).

9 L'album concept consiste à proposer une unité narrative dans la mise en relation des chansons avec un thème commun à l'album. Freak out! (1966) de Zappa and The Mothers of Invention est le premier album concept, suivi par Pet Sounds (1966) de Brian Wilson avec les Beach Boys. Mais Sgt. Pepper's Lonely Hearts Club Band (1967) des Beatles est souvent présenté comme le cas paradigmatique. Pour une discussion approfondie de l'album concept, autant pour sa portée historique que comme production musicale et produit culturel, voir Gareth Shute 2013. 
culturelle $^{10}$, soit le fait de l'inscrire dans le champ des valeurs officielles en lui accordant plus d'intérêt et de prestige que d'autres produits musicaux. Cette réalité se perçoit dans la canonisation des albums qui ont porté le genre comme Pet Sounds (1966) des Beach Boys et Revolver (1966) des Beatles (voir Jones 2008, p. 25-52), cette canonisation s'appuyant sur certains principes pour faire valoir la grandeur de ces albums : authenticité de la démarche, complexité de la musique, virtuosité instrumentale, album comme ultime accomplissement et statut élevé de l'artiste. De par la posture avant-gardiste qu'ils affichent et les références à Edgard Varèse et Igor Stravinski, Frank Zappa et ses Mothers of Invention servent de cas paradigmatique pour la mise en place de l'art rock à partir de Freak Out! (1966) (Pirenne 2011, p. 170-172). De même, le rock progressif va décliner l'ensemble des passations à l'œuvre entre la tradition classique et le rock, à commencer par la virtuosité instrumentale, les innovations formelles et harmoniques, l'élargissement de la facture instrumentale, le perfectionnement du son, etc. De nombreux exemples ont marqué les années 1970, que ce soit le "rock classique »(ibid., p. 215) d'Emerson, Lake and Palmer avec Pictures at an Exhibition (1971) ou le "rock symphonique " (ibid., p. 220) que propose le même groupe avec Brain Salad Surgery (1973). Cette époque est aussi celle qui voit les compositeurs porter un intérêt au rock, comme en font foi les musiques électroniques (par exemple, l'influence de Stockhausen chez les musiciens du Krautrock ${ }^{11}$ ) ou les réflexions de Berio (1971) à travers lesquelles le compositeur tente de cerner ce qui fait la spécificité du rock de la seconde partie des années 1960 (par exemple, les Beatles) en ciblant l'instrumentation, l'harmonie et les différences marquées avec le jazz.

Quant aux jeunes de Pink Floyd avec Sid Barrett en tête, leur premier album The Piper at the Gates of Dawn (1967) les rattache à l'art rock par la quête psychédélique et le contexte d'expérimentation sonore. Dans les années qui suivent ce premier opus, le culte du son et le statut d'artiste se nourrissent à une même recherche d'authenticité que celle qui a singularisé les avant-gardes historiques qui se sont succédé au $\mathrm{Xx}^{\mathrm{e}}$ siècle, comme en fait foi la parution de leur second album Ummagumma (1969) avec des formes de collage musical et la place colossale qu'occupent les développements instrumentaux. C'est que d'un album à l'autre, dans le passage des années 1960 aux années 1970, Pink Floyd tente d'innover en matière de sonorité en poussant toujours plus loin les techniques d'enregistrement et les formes musicales des chansons proposées, comme en témoigne le "rock orchestral » (ibid., p. 217) qu'amorce le lancement d'Atom Heart Mother (1970). Les disques qui s'enchaînent par la suite innervent chacun à leur manière les idéaux du rock progressif : le perfectionnement des techniques de studio pour Meddle (1971), l'album concept pour Dark Side of the Moon (1973), ainsi que les atmosphères instrumentales planantes pour

10 Au sens que Bourdieu donne à ce concept et qu'on retrouve à tous les niveaux dans sa théorie de la distinction culturelle (Bourdieu 1979, p. 95- 96).

11 Pour une définition du Krautrock et une discussion des principaux albums qui ont marqué le genre, voir Pirenne 2011, p. 222-226. 
Wish You Were Here (1975). Le fait de se renouveler ainsi d'un album à l'autre et de chercher à faire de chaque album une production qui se démarque, circonscrit du côté de Pink Floyd une attitude qui se rapproche de celle des avant-gardes historiques.

The Wall (1979) ne fait pas exception à ce principe d'avancée stylistique d'un album à l'autre, et en ce sens il constitue l'aboutissement de la logique avant-gardiste en proposant cette fois-ci un opéra rock sous forme de double album avec 26 chansons, à cette différence près que l'œuvre est autobiographique par la transfiguration artistique du récit de vie de Waters à travers le personnage de Pink. Dans le même souffle, elle se réalise au profit de la mise à l'écart des autres membres du groupe, sauf pour quelques chansons, par exemple le jeu de guitare de David Gilmour dans "Comfortably Numb " (voir Leroy 2009, p. 109-118). C'est ici que le lien unissant l'artiste, le double album et Montréal devient important. Comme Waters l'a expliqué à de nombreuses reprises ${ }^{12}$, le projet artistique a pris forme suite à un concert donné au Stade olympique de Montréal le 6 juillet 1977 (tournée de l'album Animals sorti en 1977) lors duquel le public s'est montré particulièrement bruyant, ce qui l'a conduit à faire monter sur scène un spectateur pour ensuite lui cracher au visage ${ }^{13}$. À ce moment de la trajectoire de Pink Floyd, le décalage entre artiste et public ne peut se faire plus évident : le travail scénographique qui sied aux effets visuels et au caractère planant des œuvres en reléguant à l'arrière-plan les musiciens, trouve difficilement sa place dans les tournées colossales où prime l'ambiance de stade (voir ibid., p. 20-22) ${ }^{14}$.

The Wall se nourrit de cette fracture entre artiste et public, Pink l'artiste se révoltant contre un public qui l'étouffe et qui est incapable de comprendre sa détresse. C'est ainsi que l'œuvre devient une mise en abyme de la célébrité rock sous fond de déchéance artistique avec en arrière-plan les rapports amour/haine, ce sur quoi va miser également Another Brick in the Wall-l'opéra. Car Pink en vient à ériger entre le public et lui un mur de manière à se protéger dans la foulée de la déliquescence psychologique qu'engendre un star système devenu étouffant. En ce sens, la pertinence de The Wall s'arrime à la réflexion générale que l'œuvre porte par rapport au rock, à la

12 Waters s'en explique ainsi dans une entrevue accordée à l'Opéra de Montréal comme outil promotionnel pour Another Brick in the Wall - l'opéra : https://www.youtube.com/watch?v=tkPlcH0NQts (consulté le 28 août 2017).

13 La performance du 6 juillet 1977 à Montréal a été enregistrée par un amateur et mise en ligne sur YouTube : https://www.youtube.com/watch?v=FJBTh0oT5HA (consulté le 28 août 2017). Dans la culture populaire, cette pratique d'enregistrement illégal se rapporte à ce qu'on appelle en anglais la pratique du bootleg. Pendant une partie d'improvisation instrumentale, plus précisément à 54 minutes et 15 secondes de l'enregistrement amateur, on peut entendre l'incident en question.

14 Il faut rappeler que durant la même période, le rock progressif entre dans un "purgatoire » (Pirenne 2011, p. 214) : à l'enthousiasme qu'a suscité le genre pendant les années 1970 et dont les ventes d'album font état, succède une période où l'on critique sa prétention et son gigantisme, voire son élitisme et son hermétisme. Par le fait même, le rock progressif va devenir la bête noire des musiciens qui veulent en revenir à un rock plus amateur et plus brute, notamment la culture punk qui l'a combattu pour son monumentalisme et sa gloire commerciale (voir Ackermann 2012, p. 5). 
performance et à sa commercialisation ${ }^{15}$; comme le suggère Ackermann, le projet de Waters met en relief 1'« interrogation intra-discursive du rock comme idéologie et pratique » (Ackermann 2012, p. 7) ${ }^{16}$.

L'art rock consolide aussi les rencontres entre opéra et rock en donnant naissance à un nouveau genre, soit l'opéra rock. L'idée revient à inscrire le rock dans un art officiel en faisant valoir une esthétique plus sérieuse tout en s'attaquant à la longue durée, bien que la performance en concert l'emporte dans plusieurs cas sur la dimension théâtrale (voir Lipscomb et Stuessy [2001]2013, p. 209) : les musiciens performent alors sur scène comme ils le feraient pour le reste de leur répertoire, à l'exception du cas où l'opéra rock est véritablement présenté dans une maison d'opéra. Mais ce qui réunit avant tout l'opéra et le rock au sein d'un même genre repose sur le sens du spectacle et l'impact du livret : l'attrait pour un drame mis en musique est la principale justification. De telle sorte que la dimension visuelle et le drame, en plus des interactions entre soliste et chœur, soutiennent cette nouvelle approche du rock. L'œuvre qui a retenu l'attention à l'époque et qui reste un modèle du genre est le Tommy de The Who, opéra de 75 minutes paru sur un album double en 1969. Fruit du travail de Pete Townshend, cet opéra met en scène un jeune garçon aveugle, sourd et muet, pourtant appelé à devenir champion de flipper ; le drame qui se joue a pour principal sujet les vicissitudes que rencontre un homme d'abord destiné à l'anonymat. L'œuvre devient une référence pour le milieu rock et The Wall en est d'une certaine façon l'héritière.

À l'instar de Tommy, The Wall propose une intrigue basée sur les états psychologiques d'un personnage : la force dramatique tient dans la succession d'événements qui sont autant d'épreuves contribuant à sa déchéance, l'opéra rock se déployant ainsi en monodrame en concentrant l'action autour du protagoniste, Tommy dans un cas, Pink dans l'autre. Mais de manière à atténuer cette dimension monodramatique qu'on retrouve souvent dans l'opéra rock, un rôle important est dévolu au chœur comme façon de susciter des interactions sociales, voire une confrontation entre le protagoniste et son environnement social. Dans The Wall par exemple, l'alternance entre soliste et chœur dans plusieurs chansons comme " Another Brick in the Wall. Part II » et « Bring the Boys Back Home », a pour effet d'affaiblir la dimension monodramatique. Dans le cas de la genèse d'Another Brick in the Wall - l'opéra, le chœur va devenir une composante essentielle comme on le verra sous peu.

15 Cette critique de ce qu'est devenu le rock par rapport aux espérances culturelles qu'il portait dans le contexte de la contre-culture des années 1960 n'est pas nouvelle en soi. Le business du rock dans le contexte d'un star-système international est pourfendu dès la fin des années 1960, et en ce sens Frank Zappa and the Mothers of Invention ont joué un rôle clé avec la parution de We're Only in It for the Money (1968) comme contre-réponse au Sgt. Pepper's Lonely Hearts Club Band (1967) des Beatles et du succès commercial qu'il a engendré (voir Wicke [1987]1995, p. 114-116).

16 "Waters had already used such feelings as inspiration for a music spectacle that stands as the most radically intra-discursive interrogation of rock as ideology and practice » (Ackermann 2012, p. 7). 


\section{LA GENĖSE D'ANOTHER BRICK IN THE WALL - L'OPÉRA}

L'intérêt que peut représenter le crossover pour l'opéra, autant dans les sujets traités que dans la musique, n'est pas en soi une nouveauté. Le $\mathrm{xx}^{\mathrm{e}}$ siècle en a offert plusieurs exemples, à commencer par les cas probants de Jonny spielt auf (1927) d'Ernst Krenek et Die Soldaten (1965) de Bernd Alois Zimmermann pour l'intégration de genres musicaux extérieurs à l'opéra - dans ces deux cas, le jazz. Plus près de nous, Greek (1988) de Mark-Anthony Turnage ressort comme un modèle du genre avec un argument portant sur un jeune skinhead (Eddy) et la misère sociale dans laquelle il baigne sous fond de violence, drogue, racisme, crise économique, bref autant de thèmes portés par la culture punk (voir Lacombe 2007, p. 220-221). Dans ce contexte, les éléments provenant du rock sont intégrés à la palette orchestrale, tout comme Fausto Romitelli ouvre An Index of Metals (2003) par un disque jouant "Shine on You Crazy Diamond" de Pink Floyd avec des effets de distorsion et de scratch ${ }^{17}$. Son vidéo-opéra pour soprano, ensemble et trois projections-vidéo, le compositeur italien l'a pensé comme une "expérience de perception totale » dans le contexte d'un " rituel laïque à la manière des light shows des années soixante, de la rave party d'aujourd'hui ${ }^{18}$ ", ce qui favorise un croisement entre opéra et culture pop. De fait, ce n'est pas seulement le rock qui a représenté un champ d'intérêt nouveau à partir des années 1980, mais plusieurs des sujets qui appartiennent à la culture populaire anglophone, comme en font foi plusieurs œuvres : le politicien californien Harvey Milk est le sujet du Harvey Milk (1995) de Stewart Wallace, l'ancienne première dame des États-Unis, Jackie Kennedy, le sujet du Jackie O (1997) de Michael Daugherty, tout comme la pop star Anna Nicole Smith le sujet d'Anna Nicole (2011) de Turnage.

Le fait que The Wall ait pu être envisagé pour un nouveau projet puis métamorphosé en opéra contemporain, s'inscrit dans les paramètres du crossover classique et de l'intérêt pour des genres et sujets dits populaires. Car la migration d'une œuvre à l'autre renforce doublement la logique du crossover: The Wall comme œuvre s'inscrivant dans les limites de l'art rock a pris forme par l'entremise de l'intérêt des musiciens rock pour la culture opératique, pour ensuite migrer vers l'intérêt des compositeurs de musique contemporaine pour l'univers rock, la boucle étant ainsi bouclée, comme on dit. Né en 1974, Bilodeau est représentatif de cette conjoncture, lui qui est arrivé à la musique par la guitare électrique ${ }^{19}$. Ce n'est pas un hasard si Romitelli l'a marqué lors d'un stage réalisé à Voix Nouvelles de l'abbaye de Royaumont en France à l'été 2003 : la guitare électrique suscite l'attention des deux compositeurs, tout comme Pink Floyd et la musique rock en général (voir McKinley 2014, p. 73-74). The Wall, c'est aussi une partie de la jeunesse de Bilodeau, comme ce l'est pour une bonne partie de sa génération.

17 L'œuvre est accessible sur YouTube et les premières mesures ne trompent pas : https://www.youtube.com/watch?v=PjxYqjyIC3E (consulté le 28 août 2017).

18 Romitelli parlant de son œuvre sur le site Internet de l'Ircam, la notice datant probablement de l'année de création de l'œuvre, soit 2003 : http://brahms.ircam.fr/works/work/11522/ (consulté le 28 août 2017).

19 Ces informations m’ont été transmises par Bilodeau lors de nos échanges. 
En ce sens, la nouveauté qu'il propose avec cet opéra est de partir d'une œuvre d'origine pour en faire une nouvelle œuvre, ce qui justifie le travail de métamorphose. Le défi était hautement risqué à la lumière de l'œuvre d'origine et de ce que Waters posait comme condition : une nouvelle ouvre certes, mais qui conserve l'argument d'origine et donc la trame narrative avec les 26 chansons du double album (voir Dufour 2017). Pour bien situer l'enjeu, les chansons fonctionnent comme autant de scènes qui jettent un éclairage, ou bien sur les blessures passées de Pink, ou bien sur les étapes de sa dégradation psychologique, ou bien sur son statut de star en lien avec son public et son entourage. Le film d'Alan Parker sorti en 1982 avec les illustrations de Gerard Scarfe fait la démonstration que «le récit [est organisé] à la manière d'un film, chaque chanson correspondant à une scène » (Leroy 2009, p. 114) ${ }^{20}$. Ainsi la question reste-t-elle entière quant à savoir où et comment poser les frontières pour créer une nouvelle œuvre. Autrement dit, comment faire du nouveau dans le cadre d'une esthétique de la revitalisation d'une œuvre rock?

Au cours des discussions avec le compositeur, ce dernier m'a avoué le casse-tête qu'a représenté la mise en place du livret avec l'obligation de respecter les mots d'origine des 26 chansons, ce qui a conduit à un livret en langue anglaise. Dès le départ, la décision a été prise de favoriser un opéra en deux actes selon la logique du double album et d'allonger la durée pour en arriver à un temps approximatif de deux heures - le double album fait 1 heure et 21 minutes. Ce faisant, les mots à la base du livret ont été travaillés par répétition et par étirement dans le temps. Sans qu'on puisse parler de transformations significatives, certains passages de l'opéra comprennent quelques ajouts de phrases de manière à convenir au drame.

À cet effet, l'ouverture est révélatrice des choix effectués par le compositeur. L'œuvre débute à l'origine par la chanson "In the Flesh? » alors que Pink donne un concert et invite les spectateurs à voir ce qu'il y a derrière ses "yeux froids " "behind these cold eyes ») : la fracture entre le public et lui est ainsi consumée en contexte de performance. Dès l'ouverture de l'opéra, la différence tient en ce que la performance se déroule au Stade Olympique de Montréal et met en scène l'événement du 6 juillet 1977 qui est à l'origine de The Wall : Waters fait monter sur scène un spectateur, puis lui crache au visage. Les mots prononcés par Waters lors de l'événement servent d'ajout au niveau textuel pour établir la communication: "You!/yes you!/lights/come on! » ${ }^{21}$, à 1'exception du mot « lights » que Pink scande dans la chanson "In the Flesh? ». L'ouverture de l'œuvre déploie ainsi un dispositif propre à la performance rock, soit des musiciens qui occupent une scène et qui affichent une attitude désinvolte face à un public déchaîné.

Cette entrée en matière est importante puisqu'elle situe ce qui est au cœur de l'opéra de Bilodeau, soit chacune des pierres comme symboles des étapes que

20 Il est à noter que la forme couplet-refrain privilégiée sur ce double album marque une rupture avec le rock progressif des albums précédents, notamment en ce qui concerne la longueur des chansons et les développements instrumentaux.

21 Voir note 13 pour écouter l'extrait. 
franchit la star du rock dans sa déchéance. C'est en misant sur ces différentes pierres que le compositeur parvient à s'éloigner du monodrame d'origine. Le protagoniste sera constamment mis en interaction avec son environnement : non seulement le public, mais aussi sa mère, son père, sa conjointe, etc. Il faut aussi ajouter la citation dans les premières mesures de l'ouverture de la Première symphonie de Brahms, une manière pour le compositeur d'établir les assises classiques de l'œuvre comme il l'a souligné en entrevue (Farber 2017) (voir la figure 1). Le fait de débuter ainsi l'œuvre mise sur la dimension " méta-rock » (Ackermann 2012, p. 11) qui est à l'origine de The Wall. Plus précisément, cette dimension méta-rock se joue sur fond de mise en perspective autoréflexive au sein de la culture rock : la performance et le statut de vedette, tout comme le poids médiatique et la relation au public, se retrouvent à être amplifiés dans Another Brick in the Wall - l'opéra. C'est que l'œuvre de Bilodeau opte pour l'approfondissement d'une mise en abyme, soit celle que propose l'ouverture : Waters à Montréal qui crache au visage d'un spectateur et entame sa déliquescence psychologique, pour enfin aboutir à une rédemption à la fois personnelle et sociale. À travers la mise à distance qu'implique le transfert du contenu narratif d'un genre à l'autre, l'opéra de Bilodeau renforce ainsi la dimension "méta-rock " contenue dans l'œuvre d'origine. Ce résultat repose sur trois choix stylistiques : le rôle du chœur, 1'approfondissement dramatique des personnages secondaires et la perspective psychanalytique recherchée.

En misant sur l'incident montréalais, le compositeur fait le choix de ne pas refermer l'œuvre sur Pink, comme c'est le cas dans le film de Parker : Pink est d'ores et déjà mis en relation avec autrui, source de son aliénation. Et avant même que Pink ne prenne la parole, le chœur affirme sa présence à travers des onomatopées : " ah » dès le premier coup d'ouverture, suivi de " hey » jusqu'au moment où Pink s'adresse à la foule (voir figure 1). Ces deux onomatopées ont pour but de marquer la présence du public dès le début ; dans "In the Flesh? », le chœur intervient plutôt au même moment que l'entrée vocale de Pink. En donnant un rôle important au chœur d'une scène à l'autre, Bilodeau crée un contexte où Pink est constamment confronté au monde qui l'entoure, le chœur étant justement cette foule à partir de laquelle l'aliénation prend forme, de telle sorte que l'écueil du monodrame est dépassé à la faveur d'éléments plus près de l'oratorio : car dans cet opéra, le chœur est partie prenante du drame autant par sa présence que par ses interventions au niveau textuel, à l'image de ce qu'on retrouve dans plusieurs œuvres de John Adams dont The Death of Klinghoffer (1991) ${ }^{22}$. Dans le cas de l'opéra de Bilodeau, l'ouverture et la fin de l'œuvre doivent être mises en rapport l'une et l'autre tant elles montrent l'évolution du chœur au sein de l'action et le rôle qu'il assume : l'œuvre s'ouvre sur une foule en liesse en contexte de performance (mise en abyme de l'événement du 6 juillet 1977), tandis qu'elle se termine avec une foule qui pardonne Pink en expliquant les raisons

22 Les parentés que partage l'opéra d'Adams avec les passions de Bach (voir Taruskin 2005, p. 522) et les oratorios de Haendel (Machart 2004, p. 113) ont été maintes fois soulignées, notamment en ce qui a trait au rôle assumé par le chœur au sein de l'action. 
d'être de l'impasse éthique et psychologique du mur, et donc qui assume un rôle vocal beaucoup plus important (voir figure 2). De par cet usage contrastant entre le début et la fin de l'œuvre, le chœur se voit confier une fonction principale au sein de l'action, étant à la fois la source de l'aliénation et l'accès à la rédemption.

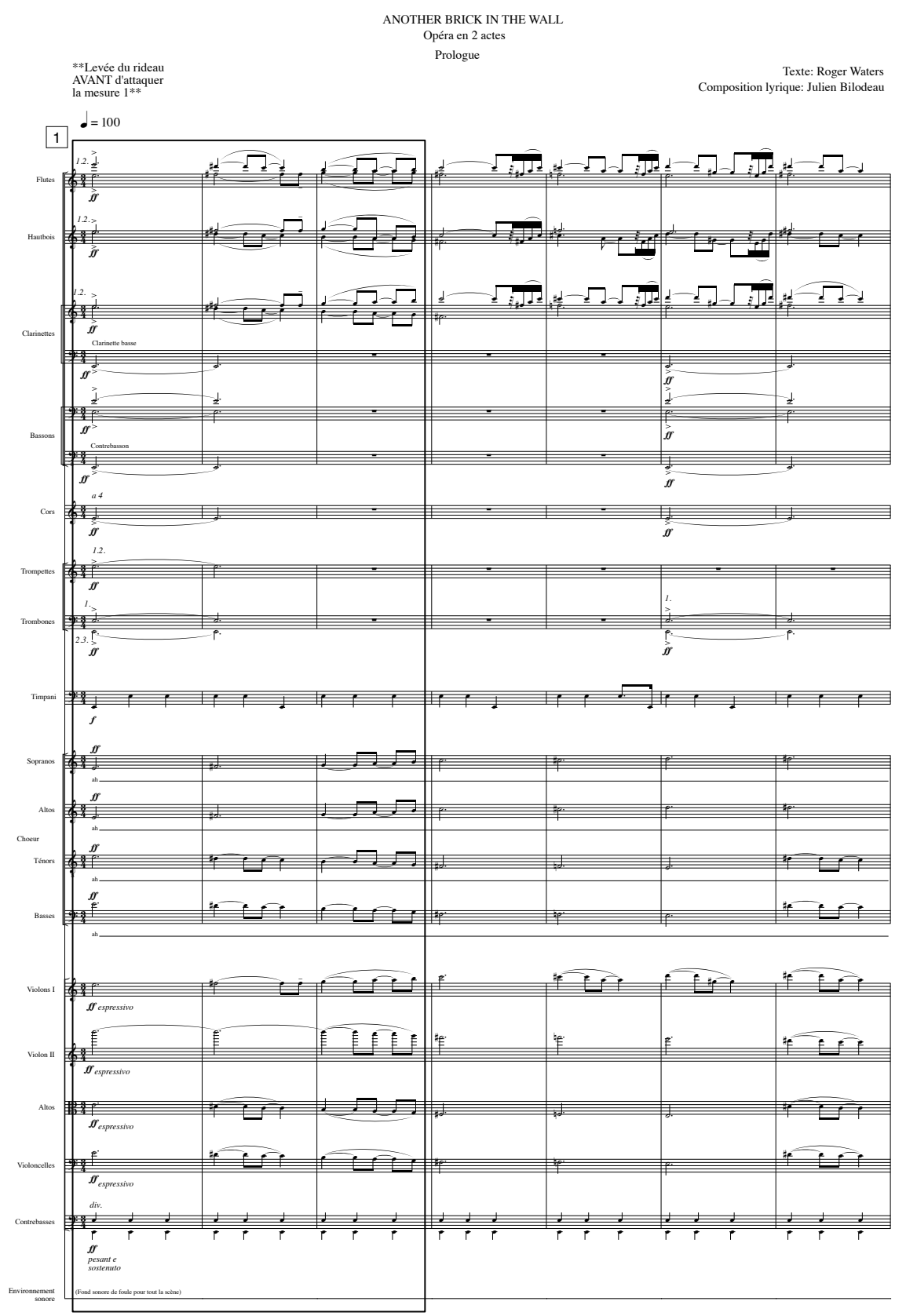

Figure 1: Julien Bilodeau, Another Brick in the Wall - l'opéra, première page. Les trois premières mesures qui se trouvent encadrées dans l'exemple sont celles où est citée l'ouverture de la Première symphonie de Brahms : on y reconnaît l'usage des timbales mais dans un rythme différent (des noires en 3/4 plutôt que des croches martelées en 6/8), le travail par rythme syncopé ainsi que les notes conjointes ascendantes en contraste avec les notes conjointes descendantes, le tout dans la tonalité de mi mineur plutôt que celle de do mineur. 

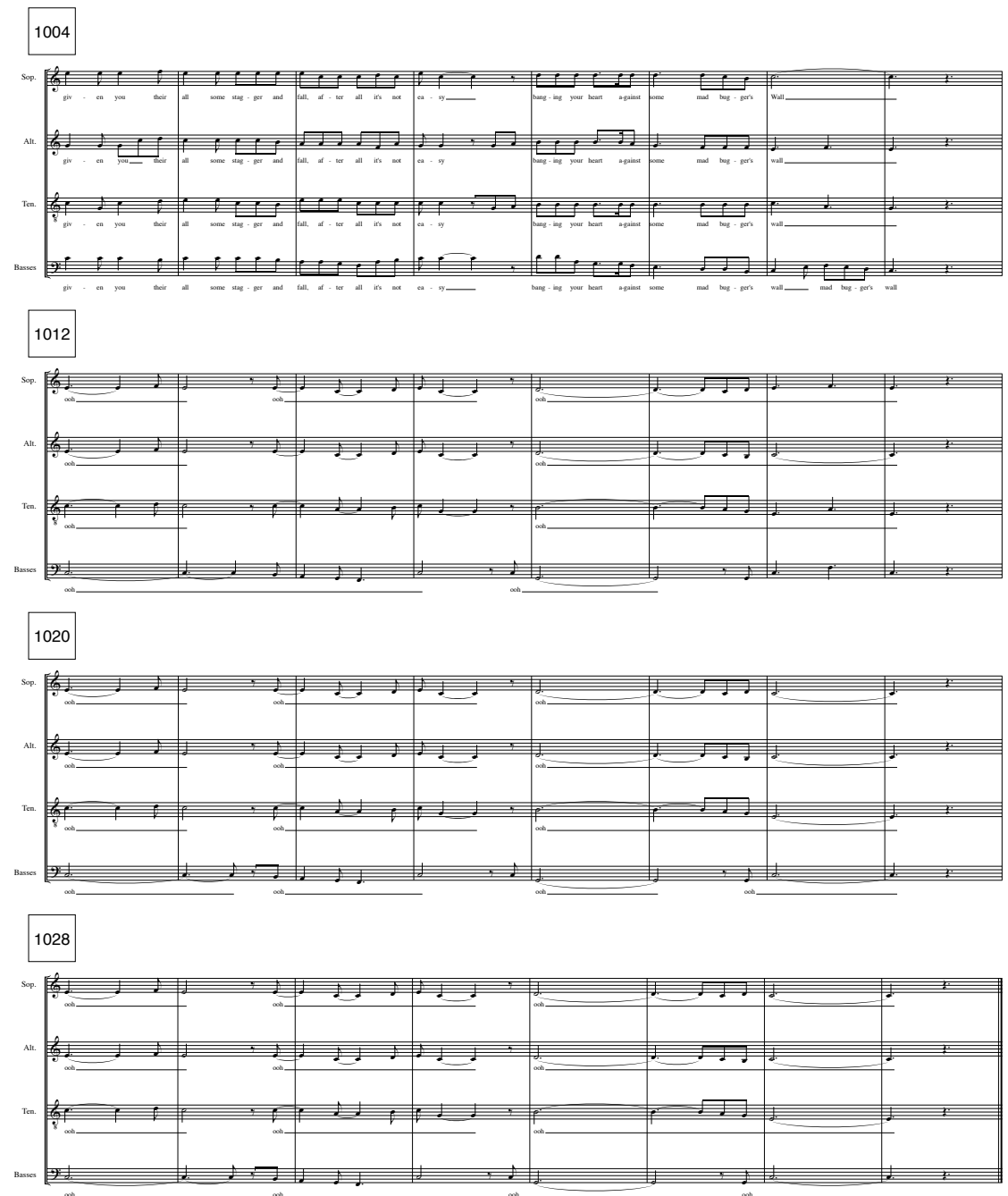

Figure 2: Julien Bilodeau, Another Brick in the Wall - 1'opéra, p. 310. Cette dernière page de la partition montre la façon dont le choeur est travaillé au sein de l'opéra et le rôle qu'il prend en fin d'ouvre: une fois les dernières paroles chantées à la mesure 1011, le choeur poursuit son chant à travers l'onomatopée "ooh" dans une harmonie de do majeur; les silences jouent un rôle de balancement entre les différentes phrases musicales tout en s'accumulant jusqu'à la fin en contraste avec les notes tenues.

La raison de la métamorphose de l'opéra rock en opéra tient aussi dans les personnages qui appartiennent à l'origine, ou bien au sous-texte (par exemple, quand Pink s'adresse à sa mère), ou bien au fond sonore utilisé dans l'enregistrement (par exemple, la présence d'une groupie dans "One of My Turns »). Bilodeau a fait le choix d'approfondir la dimension dramatique des personnages secondaires de l'œuvre d'origine, transformant ainsi certains des membres de l'entourage de Pink en personnage distinctif, comme c'est le cas pour la mère, le père et la conjointe. Ces derniers participent pleinement au tissu dramatique de l'opéra, tandis que les autres personnages, bien que leur rôle soit également approfondi, interviennent dans la scène qui justifie leur présence : ainsi le professeur, comme dans l'œuvre d'origine, apparaît-il uniquement dans les scènes "The Happiest Days of Our Lives » et "The Trial ». Par ailleurs, les rôles dévolus dans les différentes scènes sont déterminés 
par le déroulement narratif : le père et la mère assument ainsi des passages qui, à l'origine, étaient uniquement chantés par Waters et Gilmour sur 1'album, de telle sorte que les mots et la narration prennent un nouveau sens. La scène rattachée à la chanson "Goodbye Blue Sky » est caractéristique de cette situation, moment où Pink s'enlise dans son état dépressif par la réminiscence d'une enfance marquée par l'absence du père et la surprotection de la mère. Après des bruits de bombe, le chœur chante les premières paroles pour aussitôt céder la place à la mère qui chante l'entièreté de la scène, reléguant Pink à un rôle de spectateur. Le fait que la mère s'adresse à Pink situe l'action dans l'enfance et renforce ainsi l'atmosphère nostalgique de la scène. De tels développements à la fois narratifs et stylistiques par rapport à l'œuvre d'origine permettent de déployer une situation lyrique propre à l'univers opératique.

Si le monodrame est évité sur le plan formel, quelques-unes des caractéristiques du genre n'en sont pas moins renforcées sur le plan dramatique de par le récit de vie de Pink. L'opéra de Bilodeau opte pour une psychanalyse du personnage en misant sur les détresses qu'il traverse : l'incident montréalais, le retour sur les étapes marquantes de sa vie dont la perte du père et l'emprise maternelle, la déchirure avec le public, les rapports troubles avec les femmes et l'adieu au monde, constituent les moments clés de l'acte I ; la tentative d'une prise de contact avec le monde, le possible retour du père avec la présence de Vera Lynn, l'hallucination engendrée par la drogue, la folie qui conduit à la dérive fasciste, le tribunal où il demande pardon à toutes et à tous, ainsi que la fin de l'isolement comme rédemption ultime, constituent les moments clés de 1'acte II. Chacun de ces moments et la façon dont ils sont incarnés par les personnages rendent l'œuvre organique, ce que l'on peut résumer à une trame narrative qui part de la condition de star du rock, pour ensuite approfondir la psyché du personnage, pour renouer à la fin avec le monde extérieur. L'argument à la base de l'opéra prend ainsi la forme d'une radiographie des troubles de Pink selon la situation interpellée, ce qui contribue au caractère organique de l'œuvre.

Les critiques parues dans la foulée de la création ont tenté de jauger la valeur dramatique de l'œuvre par rapport à la métamorphose de l'œuvre d'origine en opéra. Entre autres, l'acte I a été davantage critiqué pour sa longueur. Christophe Huss (2017), par exemple, a insisté sur le fait que les scènes s'enchaînent dans cet acte sans qu'il soit possible d'identifier un véritable drame, d'autant qu'elles deviennent une suite d'atmosphères. En revanche, l'acte II a davantage marqué les esprits quant au drame qui se joue et la raison en est fort simple : le point paroxystique de la traversée de Pink survient à la fin de sa dérive fasciste avec la scène associée à "Waiting for the Worms ", moment où les membres du chœur subissent la violence despotique de Pink en chef de parti totalitaire (voir Grow 2017). Dans cette scène, le compositeur amplifie le volume de l'orchestre par des effets dramatiques jusqu'au point culminant, soit la juxtaposition de Pink adulte, Pink enfant et son père, Pink étant sur le point de tuer ce dernier. Le miroitement psychanalytique du personnage, qui dans l'acte I se faisait lancinant, renoue dans l'acte II avec les tourments dramatiques que l'on retrouve dans des opéras tels que Salomé, Erwartung ou Wozzeck : ce moment où le personnage, transporté dans sa folie, ne contrôle plus les gestes qu'il pose, à ceci près que Pink ne va pas jusqu'à tuer son père. 


\section{LA SYNTHÈSE STYLISTIQUE RECHERCHÉE PAR BILODEAU}

L'un des enjeux que soulève une œuvre comme Another Brick in the Wall-l'opéra est celui du rapport à l'œuvre d'origine, car si Waters a donné son accord à un nouveau projet, le risque pouvait résider dans une coupure radicale. C'est ainsi que Bilodeau a opté pour une métamorphose de l'œuvre d'origine, c'est-à-dire une absorption complète du matériau dans une nouvelle composition. Lors des discussions que j'ai eues avec le compositeur, ce dernier a comparé le matériau d'origine à des portes par lesquelles le processus de création entre et sort : quelques notes, une mélodie, un accord, bref un geste musical caractéristique de The Wall, peuvent conduire à l'invention d'un nouveau geste musical.

Dans les entrevues qu'il a accordées en amont de la création de l'opéra, Bilodeau ne s'en est nullement caché : sa démarche s'inscrit dans une tradition de l'emprunt musical où le matériau d'une œuvre préexistante est insufflé d'un nouveau sens, citant au passage Bach par rapport à Vivaldi et Brahms par rapport à Beethoven ${ }^{23}$. Qu'en est-il dans le cas d'Another Brick in the Wall - l'opéra ? La métamorphose recherchée fait intervenir le matériau de base à travers la logique des portes à enfoncer, mais pour aussitôt lui donner un nouveau souffle musical. Si l'on reconnaît parfois le matériau de Waters, surtout les contours mélodiques et les progressions harmoniques, ceux-ci sont toujours absorbés dans de nouvelles harmonies, de nouveaux rythmes, en plus de la texture instrumentale et du travail d'orchestration.

Les seules exceptions notables à cet effet sont les deux scènes de l'acte II où le compositeur s'est contenté d'orchestrer la musique d'origine, de telle sorte qu'on peut parler ici d'arrangement musical. Dans la scène "Vera ", la chanson "We'll Meet Again » de Vera Lynn est intégrée à l'opéra dans une orchestration à mi-chemin entre le style big band et l'orchestre jazz à la Benny Goodman ${ }^{24}$, suite à quoi une section effectue une transition vers "Brings the Boys Back Home ", qui à l'origine avait été orchestrée par Michael Kamen - les arrangements orchestraux dans The Wall sont le fruit de son travail. Bilodeau a conservé l'essentiel du travail de Kamen pour "Brings The Boys Back Home », les différences étant du côté des ajustements instrumentaux et de l'intégration des registres vocaux pour les personnages (soit Pink, la mère, le père et Vera chantant avec le chœur). L'autre scène que le compositeur s'est contenté d'adapter en préservant la musique d'origine est celle du jugement avec "The Trial » : l'orchestration de Kamen a subi à nouveau des ajustements pour les instruments, les textures orchestrales, ainsi que les parties vocales. Le compositeur s'en est justifié dans nos échanges en insistant sur le caractère théâtral de la chanson. En effet, «The Trial » possède une dimension opératique dans sa facture d'origine en

23 L'entrevue a été réalisée par l'Opéra de Montréal comme outil promotionnel dans le contexte de la création de l'opéra de Bilodeau : https://www.youtube.com/watch?v=LiXT6Kp8weo (consulté le 28 août 2017).

24 En ce qui concerne le double album de 1979, la chanson de Vera Lynn est mentionnée dans «Vera », mais n'est pas jouée. Elle est seulement citée au niveau textuel dans les paroles chantées par Pink:

"Remember how she said that/We would meet again». 
se rapprochant des opéras du duo Brecht-Weill (observation de Bilodeau lors de nos entretiens). Dans le cas de ces deux scènes de l'acte II, la métamorphose cède le pas à un arrangement et à des transformations strictement formelles.

Les autres chansons situent davantage la métamorphose à la base de l'opéra : le matériau d'origine s'infiltre ici et là et est travaillé dans une nouvelle facture. Le cas de la chanson "Hey You ", si caractéristique du double album, donne un bon aperçu du travail de Bilodeau. De la progression harmonique de la chanson d'origine $^{25}$, Bilodeau conserve l'alternance des deux accords mineurs à distance d'un ton descendant (couleur phrygienne), mais pour en faire dans chaque cas une collection (transposé en sol dièse) de six notes en mineur harmonique, la sensible en moins : l'accord de mi mineur avec neuvième ajoutée qui ouvre la chanson d'origine donne ainsi une première collection de notes (sol\#-la\#-si-do\#-ré\#-fa\#), tandis que l'accord de ré mineur avec neuvième ajoutée, toujours de la chanson d'origine, donne une seconde collection de notes (fa\#-sol\#-la-si-do\#-mi, voir figure 3). L'alternance de ces deux accords pose ainsi le matériau de base de la scène et se perçoit à tous les niveaux, par exemple dans l'alternance entre le chœur et le père au début de la scène. Si l'on perçoit à l'oreille le cadre harmonique d'origine avec l'alternance des deux accords, entre autres la couleur phrygienne, le travail de Bilodeau rend néanmoins le passage plus ambigu au niveau harmonique puisqu'il s'adonne en quelque sorte à un travail de brouillage : des mouvements conjoints viennent ainsi se superposer aux notes principales des deux accords en mineur, entre autres le mouvement brodé de la partie vocale assumée par le père autour de la note la dièse et les ostinati aux cordes. De telle sorte que le résultat dans cet exemple précis est celui d'une fusion stylistique entre la progression harmonique d'origine et les nouvelles combinaisons orchestrales, harmoniques et rythmiques travaillées par Bilodeau.

La constitution de l'orchestre donne également une bonne idée des choix effectués par le compositeur : une timbale fait office de seule percussion et un synthétiseur s'ajoute à la palette orchestrale. Le synthétiseur est pour sa part confiné à un rôle de renforcement orchestral avec la tenue de notes ou d'accords ${ }^{26}$. Mais ce qui contribue à mettre l'orchestre au service du drame est le rôle conféré aux cordes. Ces dernières remplissent une fonction lyrique, à l'image de ce qu'on retrouve dans les opéras d'Adams comme Nixon in China (1987) et The Death of Klinghoffer (1991). Entre autres, la dimension lyrique dans les opéras d'Adams a souvent été résumée par la façon dont le compositeur recherche des climax émotionnels en travaillant le timbre et la masse sonore des cordes (entre autres par des cycles en tierces majeures

25 La chanson s'ouvre sur l'alternance de deux accords arpégés, soit mi mineure avec neuvième ajoutée et ré mineure avec neuvième ajoutée, conférant ainsi une couleur phrygienne au passage avec les deux accords mineurs à distance d'un ton descendant. Une fois les paroles entamées, l'harmonie s'affirme clairement en mi mineur naturel (mode éolien) avec l'alternance du premier degré (mi mineur) et du cinquième degré (si mineur), suite à quoi l'harmonie module au relatif majeur, soit la tonalité de sol majeur avec la progression V-I-V-IV, l'accord de si mineur jouant le rôle de pivot d'une tonalité à l'autre.

26 Comme il a été difficile de percevoir cet instrument en salle, le compositeur a avoué lors de nos échanges que le résultat obtenu par cet ajout n'est pas concluant. 
ou en tierces mineures) par l'accentuation de notes, les envolées en crescendo et les effets d'apothéose (Taruskin 2005, p. 519). Une situation semblable est à 1'œuvre dans l'opéra de Bilodeau. La scène rattachée à « The Thin Ice (1)» montre le rôle qu'il assigne aux cordes en remplissant l'espace harmonique et en contribuant à la masse orchestrale par des effets de volume et d'amplification, travaillant ainsi dans une dimension plus près de la musique symphonique. C'est que le compositeur cherche aussi à dramatiser son orchestre par l'amplification de la texture : l'orchestration joue régulièrement sur les effets de contraste entre les textures instrumentales et sur les effets d'apothéose que permettent les crescendo et les tutti, comme dans la musique symphonique du XIX ${ }^{\mathrm{e}}$ siècle, qu'on pense à Berlioz, Liszt ou Mahler ${ }^{27}$.

Les parallèles avec les opéras du compositeur américain ne s'arrêtent pas là. Car par le travail accordé à la répétition dans le choix des motifs, figures et gestes, Bilodeau amarre sa musique à un style post-minimaliste, soit un style qui, chez Adams, " exige une articulation des textures ou des harmonies beaucoup plus rapides » et " qui se complaît dans un éventail "impur" de possibilités stylistiques " (RamautChevassus 2003, p. 107), ce que suppose d'une certaine façon la métaphore des portes à enfoncer dans la métamorphose du double album en opéra. Et cette parenté ne repose pas uniquement sur la citation dans « Empty Spaces » d'un motif mélodico-rythmique provenant de Music for 18 Musicians (1976), l'une des œuvres phares de Steve Reich. Les premiers violons dans cette scène évoluent par figures répétitives en tournant autour des mêmes notes et en conservant le même rythme de double-croches, ce qui donne pour résultat une musique près de celle que l'on retrouve chez Reich et Adams. C'est aussi le cas dans la scène de "Young Lust » où les gestes musicaux employés à l'orchestre prennent en quelque sorte une configuration archétypale (voir figure 4). De même, aux endroits où l'action avance de manière soutenue comme dans les trois scènes de "Another Brick in the Wall ", les figures rythmiques sont travaillées à la fois par répétition soutenue, par addition instrumentale et par évolution harmonique (voir figure 5). La figure 5 montre ce qu'on retrouve dans plusieurs passages de l'opéra de Bilodeau : l'emploi d'accords de quatre ou cinq sons par ajout graduel sous forme évolutive pour chacune des notes de l'accord, par exemple un motif qui place deux notes de l'accord puis deux autres motifs qui, en superposition, viennent compléter l'accord, mais tout cela à l'intérieur d'un continuum musical que permet le travail par figures répétitives.

27 Comme plusieurs compositeurs québécois nés dans les années 1960 et 1970, Bilodeau a fait de l'orchestre un outil significatif dans le développement de sa poïétique et de sa carrière, et en ce sens son opéra témoigne de l'expérience qu'il a acquise dans le domaine symphonique (voir Trottier 2017, p. 35-36). 
24
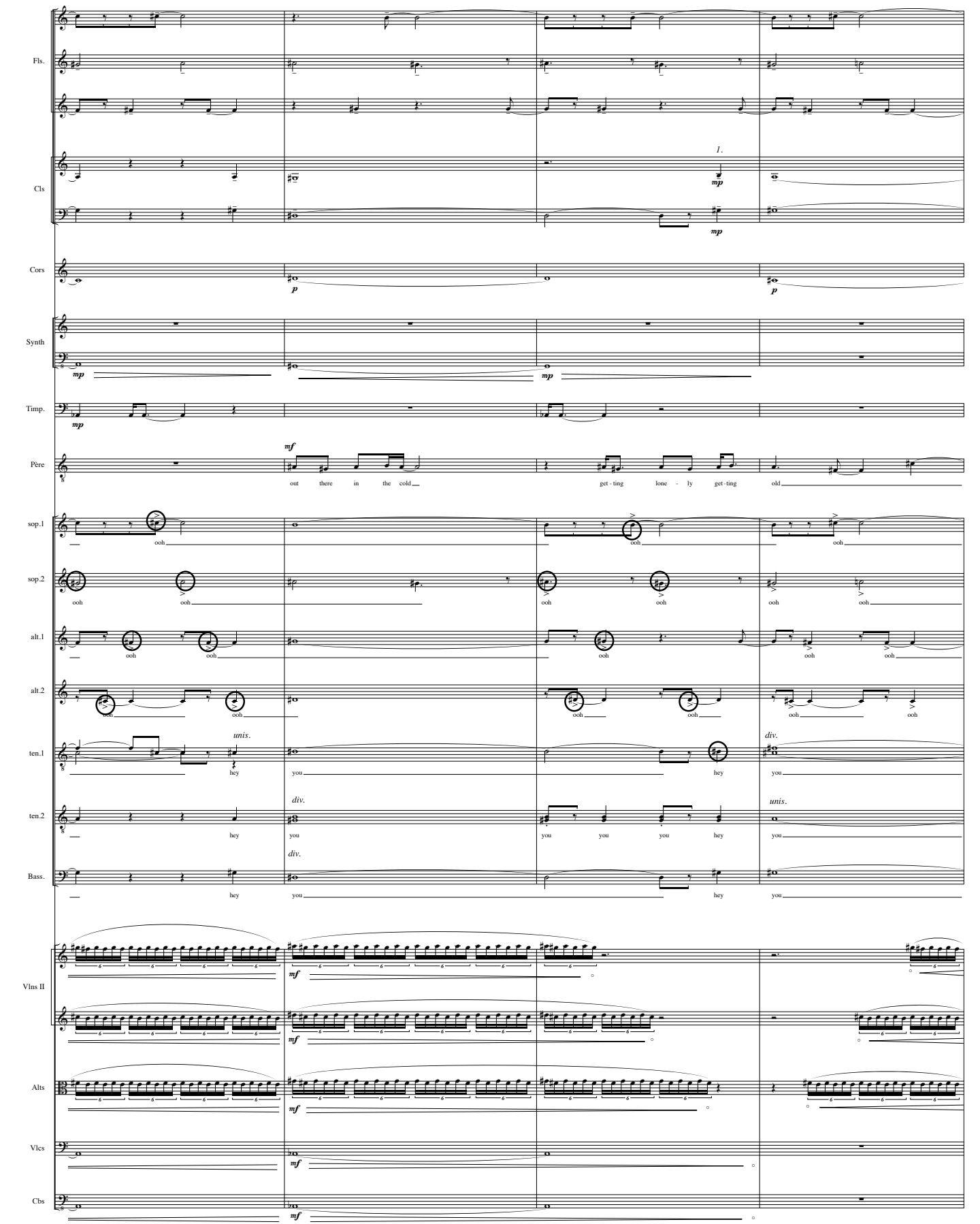

Figure 3 : Julien Bilodeau, Another Brick in the Wall - 1'opéra, p. 176. Les notes entourées dans les lignes vocales chantées par le choeur sont les quatre principales notes des deux accords, soit fa dièse mineur avec la neuvième ajoutée pour la première et dernière mesures et sol dièse mineur avec la neuvième ajoutée pour les deux mesures du milieu. 


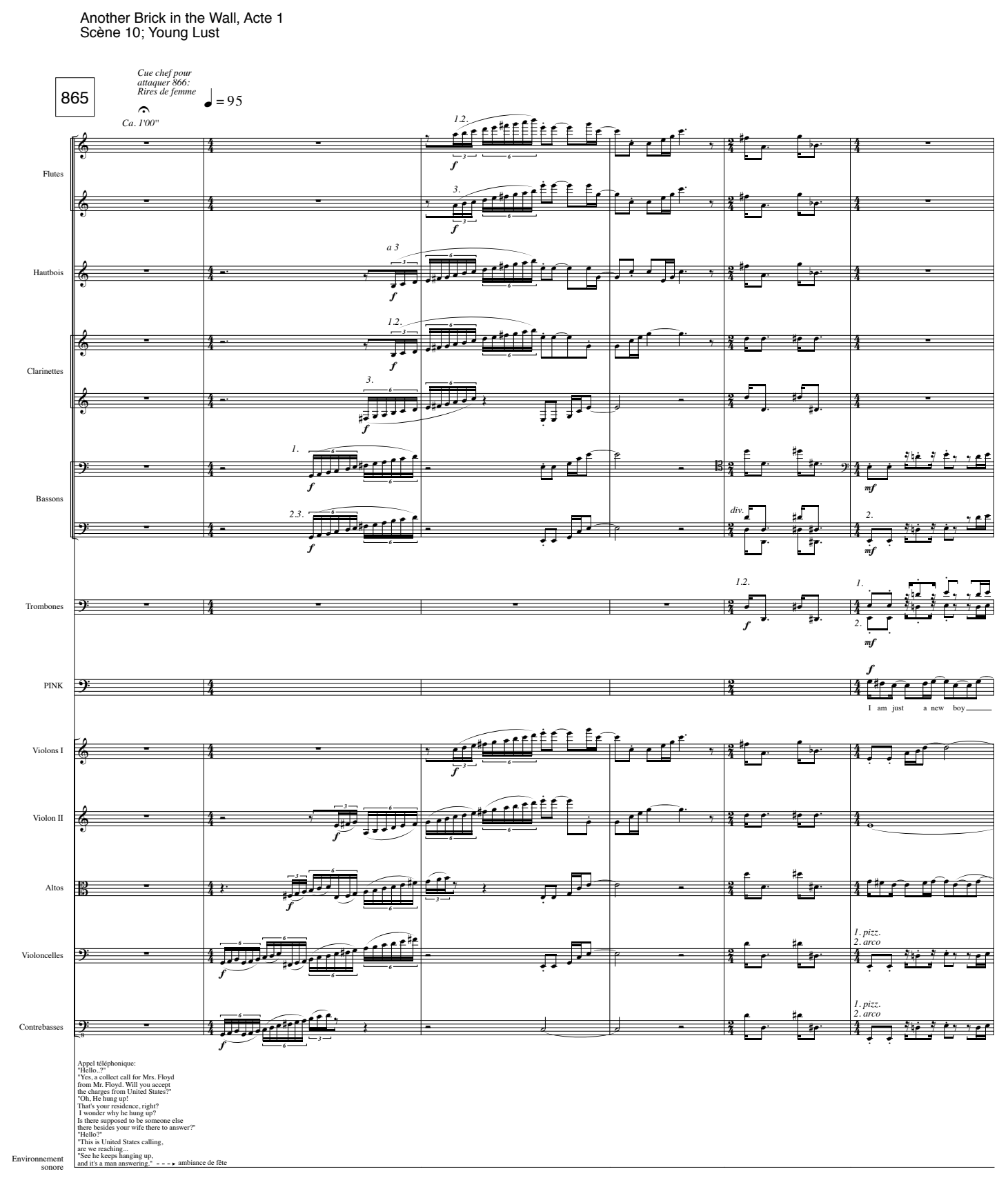

Figure 4: Julien Bilodeau, Another Brick in the Wall - 1'opéra, p. 121. Dans ces six premières mesures de la scène associée à la chanson "Young Lust", on peut voir à l'oeuvre le travail de Bilodeau à travers une figure archétypale : le motif évoluant du grave à l'aigu par mouvement conjoint ascendant est relayé d'un instrument à l'autre (des contrebasses jusqu'aux flûtes) et devient ainsi le geste phare qui campe l'atmosphère de la scène. 


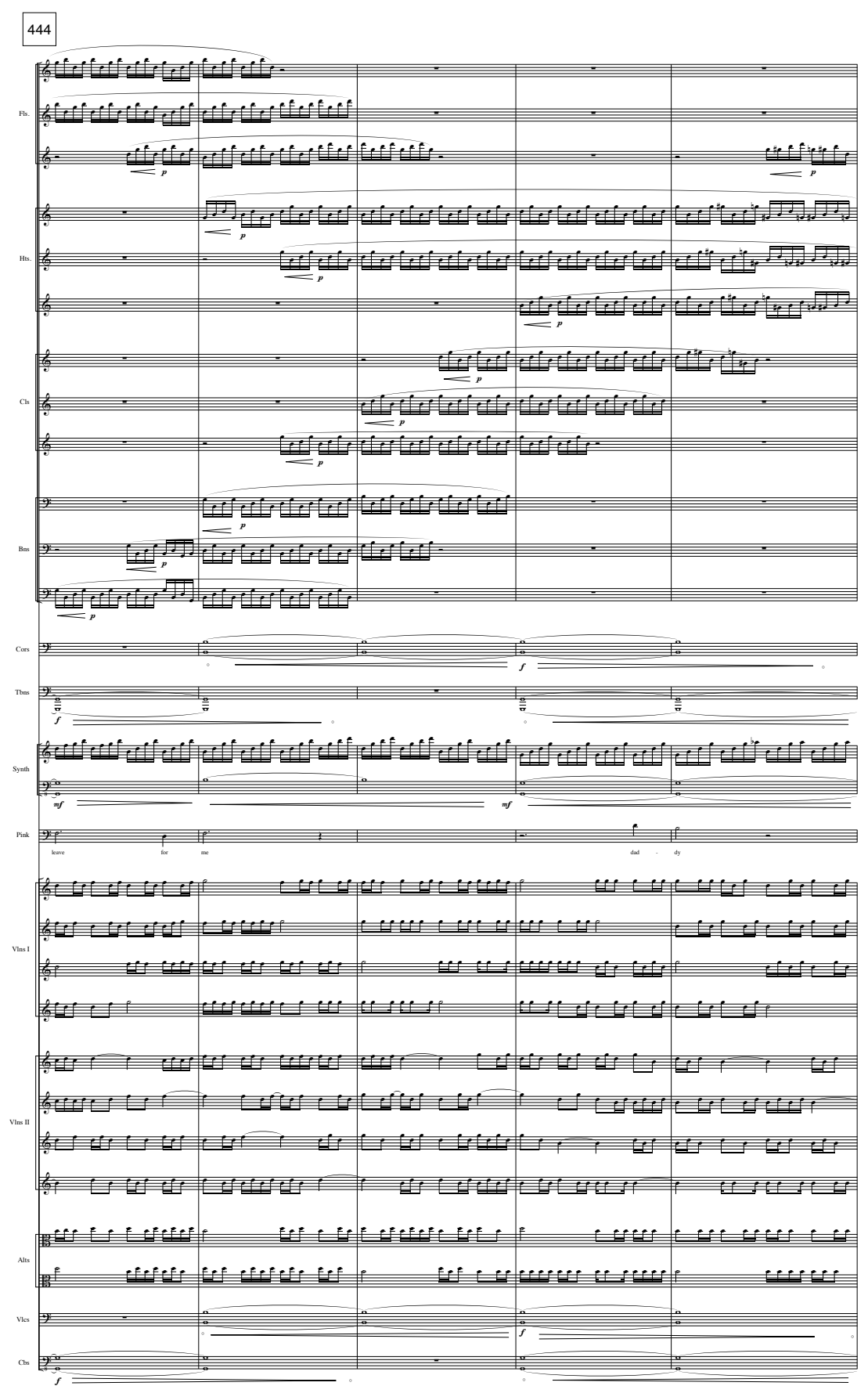

Figure 5 : Julien Bilodeau, Another Brick in the Wall - l'opéra, p. 53. Cette page tirée de la scène rattachée à la chanson "Another Brick in the Wall. Part I" donne un bon aperçu des figures en répétition qu'utilise Bilodeau et la manière dont il les travaille par section instrumentale en créant des effets d'addition ou de soustraction dans la texture d'ensemble.

Dans ce contexte, quel style adopte Bilodeau dans Another Brick in the Wall-l'opéra? Le style global de l'œuvre ne saurait être qualifié uniquement de post-minimaliste. La formation de Bilodeau l'a conduit à travailler avec des compositeurs aussi différents que Stockhausen, Pascal Dusapin et Romitelli. Ce dernier a exercé une profonde influence sur lui, ce que résument les paroles prononcées par le compositeur italien 
en sa présence et qu'il a rapportées dans une entrevue accordée à la revue Circuit, musiques contemporaines : "Vous savez, je crois que votre musique, si savante qu'elle puisse être, ne doit jamais manquer à votre désir." Voilà le noyau, le cœur, le centre autour duquel tout est désormais possible » (Bilodeau, cité par McKinley 2014, p. 74). Bilodeau s'accapare d'une certaine façon la liberté stylistique dont se revendique Romitelli en faisant "voler en éclats les deux sphères du high art et du low art " (Lacombe 2007, p. 267) : l'emprunt et l'usage de matériaux et de techniques extrinsèques à la tradition classique, mais toujours au service d'une expression hautement personnalisée - là encore des parallèles peuvent être dressés avec l'attitude d'Adams, notamment par l'ouverture aux musiques populaires (RamautChevassus 2003, p. 110). Bilodeau en vient ainsi à travailler à partir d'un « coffre à outils étendu » (Gonneville 2012, p. 107), non pas tant pour interpeller la tradition musicale ou pour faciliter la communication que pour s'approprier de façon personnelle des techniques ou des manières de faire éprouvées.

En somme, le travail de Bilodeau dans cet opéra résulte en une synthèse stylistique. Les associations qui se créent à l'oreille n'en sont pas moins importantes et doivent être soulignées à grand trait, que ce soit l'influence des musiques minimalistes, les références à Brahms et Reich ou les mélodies et harmonies provenant des chansons de The Wall. L'œuvre fonctionne donc à travers des repères stylistiques qui sont autant d'éléments musicaux qui viennent tisser une toile de significations à l'écoute. De telle sorte que cet opéra s'inscrit dans l'approche cumulative qu'a théorisée Hervé Lacombe pour l'opéra du $\mathrm{xx}^{\mathrm{e}}$ siècle, soit une approche qui " englobe tous les temps " et " considère, en un vaste mouvement rétrospectif, sans hiérarchie et sans chronologie, l'éventail des possibles et matériaux déposés par chaque époque » (Lacombe 2007, p. 74). Bilodeau, de par la fusion stylistique à laquelle il s'adonne, le choix d'un orchestre mis au service du drame et la place importante conférée au chœur, poursuit cette approche cumulative. Son opéra, autant par l'œuvre préexistante que par la tradition musicale qui le précède, est impensable en dehors des limites d'une approche qui se veut cumulative, et peut-être consensuelle en prenant une distance par rapport aux innovations technologiques et vocales mises en valeur dans le théâtre musical et dans plusieurs œuvres de musique contemporaine centrées sur la voix (voir Salzman et Desi 2008, p. 19-29).

\section{LE POIDS DES CONVENTIONS RATTACHÉES À L'OPÉRA ET AU ROCK}

Si la métamorphose à laquelle le compositeur s'est adonné dans le passage de l'œuvre d'origine à Another Brick in the Wall - l'opéra retient 1'attention, il en va tout autant pour les conventions qui sont en jeu dans la rencontre entre opéra et rock. Car il faut aussi jauger les impacts qu'engendre le passage d'une œuvre à l'autre du côté de la réception, The Wall faisant partie du canon de la musique rock $^{28}$. Le crossover

28 L'album se retrouve ainsi dans plusieurs des compilations des meilleurs albums rock, ces listes étant à la base de la canonisation du rock ces dernières décennies (voir Jones 2008). Dans le cas de The Wall, pour ne donner qu'un exemple, l'album est arrivé au 87e rang du classement effectué en 2003 par le magazine 
entre opéra et rock s'établit dans le cas d'Another Brick in the Wall-l'opéra au sein d'une institution qui accueille de manière générale des opéras et qui est donc étrangère au contexte de diffusion des musiques rock en situation de concert. La métamorphose de l'œuvre d'origine, même si elle est totalement réussie, ne prédispose pas nécessairement à un accueil favorable du public, étant donné la relation que chacun entretient avec The Wall et les distances qui séparent le monde du rock de celui des musiques dites classiques. C'est ainsi que plusieurs embûches peuvent se dresser dans ce croisement entre opéra et rock, entre autres du côté des conventions propres à la fois aux genres convoqués et à la rencontre de traditions artistiques différentes. Nonobstant les enjeux stylistiques évoqués plus haut dans la métamorphose de l'œuvre, les implications du crossover classique semblent nettement plus problématiques du côté de la réception. Et cela tient en partie au contexte particulier de l'opéra ${ }^{29}$.

C'est ainsi qu'Another Brick in the Wall - l'opéra peut servir de cas paradigmatique pour éclairer ce qui est en jeu du côté des conventions à la lumière des difficultés et défis qui parsèment la réception du crossover classique, les représentations montréalaises ayant clairement mis en évidence cette réalité. Comme les organisateurs tout autant que les critiques musicaux l'ont souligné (파ber 2017 ; Grow 2017 ; Houle 2017), le public se déclinait à la faveur de deux horizons, soit d'un côté la culture rock, de l'autre la culture opératique. Malgré le fait que l'opéra se voulait une métamorphose de l'œuvre d'origine en mettant en valeur le travail de Bilodeau, la campagne publicitaire de l'Opéra de Montréal a largement misé sur la popularité de The Wall et sur son lien avec Montréal, créant ainsi toutes sortes d'attente. Par exemple, lors des représentations, un kiosque placé tout juste à côté des vestiaires exhibait plusieurs articles à vendre, comme dans un concert rock : macarons, chandails et autres artefacts reprenaient les images des affiches publicitaires et étaient vendus à titre d'accessoires promotionnels. Lors de la première de l'œuvre, comme cela se voit en situation de concert rock, certains spectateurs faisaient la file pour pouvoir se procurer l'un des objets publicitaires rattachés à l'œuvre. De même, les images utilisées autant que les discours et slogans mélangeaient à tous les niveaux l'œuvre d'origine et la nouvelle œuvre ${ }^{30}$.

(hautement influent dans le domaine du rock !) Rolling Stone, sous le nom de Rolling Stone's 500 Greatest Albums of All Time: http://www.rollingstone.com/music/lists/500-greatest-albums-of-all-time-20120531/ pink-floyd-the-wall-20120524 (consulté le 28 août 2017).

29 Les réflexions esthétiques et sociologiques sur l'opéra ont souvent porté sur le poids de la tradition au regard du répertoire consacré et sur la difficulté d'innover en matière de création artistique, comme en fait foi le célèbre article de Theodor W. Adorno paru en 1962 et publié dans une traduction française en 1985. Il est à noter toutefois que cet écrit d'Adorno est paru avant que les compositeurs associés à la musique dite contemporaine n'investissent le genre opératique, par exemple Die Soldaten (1965) de Zimmermann.

30 La bande annonce réalisée par l'Opéra de Montréal comme outil de vente illustre bien le mélange des genres auquel on a assisté et l'imaginaire rock qui a été mis en valeur : https://www.youtube.com/ watch?v=UoxG9aqlngg (consulté le 28 août 2017). 
La conséquence directe de ces mélanges entre opéra et rock est que les deux types de public ne partageaient pas le même horizon d'attente ${ }^{31}$, cette division s'étant reproduite dans les recensions critiques publiées par les quotidiens montréalais. Et à travers les médias sociaux, certaines personnes ont reproché à l'œuvre sa complexité et sa rupture avec l'œuvre d'origine ${ }^{32}$. L'horizon d'attente est donc un facteur non négligeable ici : pour les amateurs de rock, il était de manière générale dirigé vers une adaptation symphonique de The Wall, ou à tout le moins une reconnaissance plus claire des mélodies des 26 chansons $^{33}$, tandis que pour les amateurs d'opéra, 1'horizon était dirigé dans les limites de l'opéra contemporain, ou à tout le moins de la tradition opératique. Le crossover à la base de l'œuvre allait donc forcément faire des mécontents d'un côté comme de l'autre, et c'est ce qui est arrivé chez plusieurs amateurs de Pink Floyd. Lors de la première du 11 mars 2017, j'ai pu constater après quelques minutes que certains spectateurs quittaient la salle ; et après l'entracte, on pouvait apercevoir quelques sièges vides. Or chez les critiques de musique populaire et rock, à l'exception du journal Le Devoir avec une critique virulente de l'œuvre par Sylvain Cormier (2017) à travers l'image choque " frapper un mur ", l'œuvre a été bien accueillie : c'est le cas de Nicolas Houle (2017) au Soleil, de Brendan Kelly (2017) à Montreal Gazette et aux critiques provenant de l'international comme Kory Grow (2017) du Rolling Stone. Certains n'en ont pas moins critiqué, à l'instar de Huss (2017), la longueur du premier acte par rapport à l'efficacité du second, par exemple Odile Tremblay (2017) du Devoir et Caroline Rodgers (2017) de La Presse. Chez certains critiques, l'œuvre proposée par Bilodeau a été jugée comme un renouvellement positif de The Wall, l'opéra venant ainsi bonifier le double album par les nouvelles dimensions dramatiques qu'il a permis de développer. C'est ainsi que Jim Farber (2017) de l'Observer a parlé d'un opéra pertinent, alors qu'Alain de Repentigny (2017) dans La Presse a insisté sur " une relecture utile ».

Comme la plupart des critiques l'ont souligné, et comme la couverture médiatique en a fait la démonstration en naviguant du classique au rock, Another Brick in the Wall - l'opéra s'adressait à des publics d'horizons différents et c'est ainsi qu'est intervenu l'enjeu des conventions. Car les conventions propres au rock ne sont pas celles propres à l'opéra, autant du côté de la production que du côté de la réception : les deux genres se déploient dans des horizons musicaux, culturels, esthétiques, sociaux et économiques différents, comme le montre à voir leur histoire respective. Comme l'explique Howard S. Becker dans Les mondes de l'art ([1982]1988), pour qu'un

31 Ce concept est emprunté à la théorie esthétique d'Hans Robert Jauss ([1972]1978). Ce que dit Jauss de la réception de l'œuvre littéraire peut s'appliquer à l'opéra de Bilodeau dans le rapport à son public au regard de The Wall : «La façon dont une œuvre littéraire, au moment où elle apparaît, répond à l'attente de son premier public, la dépasse, la déçoit ou la contredit, fournit évidemment un critère pour le jugement de sa valeur esthétique » (Jauss [1972]1978, p. 58).

32 Entre autres, la prise de position d'une chroniqueuse montréalaise rattachée au Journal de Montréal, soit Sophie Durocher (2017), a aussi fait sensation avec le titre : " The Wall sans The Wall, c'est pas The Wall».

33 Lors de la première du 11 mars 2017, la présence de Waters a été soulignée par les organisateurs avant la levée du rideau, ce qui a donné lieu à une ovation qui était plus digne du début d'un concert rock que du début d'un opéra. Certains amateurs de rock criaient le nom de Waters pendant l'ovation. 
monde de l'art advienne et que le travail artistique se coordonne de façon maximale, des procédures, c'est-à-dire des manières de faire qui sont assimilées par les individus, doivent s'installer. Les conventions occupent une fonction tout aussi centrale dans la réception puisqu'elles permettent une communication maximale entre l'artiste et le public (Becker [1982]1988, p. 53-58), notamment à travers la maîtrise des codes de base dans un genre donné de manière à recevoir et à déchiffrer les œuvres qui y sont associées, par exemple à l'opéra. Le rapport à la performance et le type d'écoute et de réactions qu'engendre la situation de concert, en sont des exemples éloquents dans les comparaisons entre opéra et rock : d'un côté, le concert classique et l'opéra se déploient de manière normalisée avec une forme de distance pour maximiser la situation d'écoute; de l'autre, le concert rock permet l'expression des réactions les plus spontanées et une relation plus directe avec les artistes - Antoine Hennion a analysé la différence entre les deux situations de concert et a parlé d' " emprise de la musique » dans le cas de la performance classique et de "scène primitive » dans le cas de la performance rock (Hennion 1993, p. 324-326 et p. 335-339).

Dans le cas de l'opéra de Bilodeau, on peut émettre l'hypothèse qu'une partie du public ne possédait pas les codes nécessaires à la compréhension de l'œuvre, soit les conventions rattachées à l'opéra : le drame mis en musique, la mise en scène, la durée de l'œuvre, le travail vocal, l'alliage entre orchestre et voix, sont des dimensions qui pouvaient ne pas faire partie des habitudes acquises en terme d'écoute. Dans ce contexte, il était difficile pour ce public de comprendre l'importance accordée au drame dans la métamorphose de l'œuvre et la synthèse stylistique à la base du langage musical, ce qui peut conduire à une forme de malentendu culturel (Pedler 2004) : les jugements sévères exprimés par les critiques mentionnés plus haut, entre autres Cormier (2017) et Durocher (2017), vont dans le sens de ce malentendu. En effet, leur jugement achoppe sur le fait que The Wall était méconnaissable et que l'œuvre de Pink Floyd a été ainsi dénaturée, ce dont témoigne bien le titre "The Wall sans The Wall, c'est pas The Wall » (ibid.). À l'inverse, on peut aussi émettre l'hypothèse selon laquelle certains amateurs qui maîtrisaient les conventions propres à la tradition opératique, et qui plus est l'opéra contemporain, n'ont pas apprécié l'œuvre de Bilodeau, les raisons pouvant alors être nombreuses.

Mais un fait demeure et il était perceptible autant lors de la première que dans la couverture médiatique qui s'en est suivie : les conventions propres à la performance rock et à l'histoire du genre sont entrées en collision avec les conventions institutionnelles propres à une maison d'opéra. Certains amateurs rock et certains critiques, visiblement peu préparés à ce genre de métamorphose d'un album en opéra, se sont demandés dans quel but et à quel prix on détournait ainsi l'œuvre qu'ils admirent. Car il y a une valeur qui s'est imposée dans la canonisation que le rock a connue ces dernières décennies et qui transforme des œuvres comme The Wall en objets sacrés : le respect de l'œuvre d'origine, autant pour la valeur qu'elle porte que pour le plaisir à la réécouter (voir Jones 2008, p. 42-52). Ainsi certains amateurs de rock en sont-ils venus à reprocher à l'opéra de Bilodeau ce qui est au fondement même de l'institution opératique dans laquelle ils se trouvaient : le respect, voire la sacralisation d'une œuvre. 


\section{CONCLUSION}

Les implications du crossover entre opéra et rock doivent donc tenir compte autant des difficultés que suppose du côté poḯtique la métamorphose d'une œuvre à l'autre que le malentendu, voire le choc du côté esthésique que peuvent engendrer des conventions différentes d'un genre à l'autre. La création d'Another Brick in the Wall - l'opéra de Bilodeau en mars 2017 aura été l'occasion à Montréal d'observer ce qui se joue dans ce genre de production et de jauger ce que différents publics y gagnent, notamment les publics respectifs de l'opéra et du rock. Car si à terme la métamorphose d'un album rock en opéra peut s'appuyer sur quelques exemples de crossover classique qui ont marqué les esprits comme Tommy du côté rock ou Greek du côté de l'opéra, chaque production musicale qui mise sur le crossover classique s'aventure aussi en terrain inconnu par rapport aux conventions qui sont en jeu et aux réactions que susciteront les comparaisons auxquelles se livreront les publics respectifs. C'est un jeu qui en vaut pourtant la chandelle et qui peut donner lieu à des relectures substantielles et pertinentes d'œuvres issues d'horizons musicaux différents, ce dont a témoigné le travail réalisé par Bilodeau et toute l'équipe de production derrière Another Brick in the Wall - l'opéra. Il reste à voir si les maisons d'opéra, déjà frileuses à programmer de nouvelles œuvres, accepteront dans les prochaines années de se lancer dans des productions de ce genre considérant les défis à relever.

\section{BIBLIOGRAPHIE}

Ackermann, Zeno (2012), " Rocking the Culture Industry/Performing Breakdown. Pink Floyd's The Wall and the Termination of the Postwar Era ", Popular Music and Society, vol. 35, n 1, p. 1-23.

Adorno, Theodor W. ([1962]1985), «Opéra », Contrechamps, n 4, p. 6-17.

« An Index of Metals (2003)», http://brahms.ircam.fr/works/work/11522/, consulté le 20 mars 2017.

Beaucage, Réjean (2004), « Frank Zappa dans l'univers des musiques sérieuses. Un parfait étranger?», Circuit, musiques contemporaines, vol. 14, $\mathrm{n}^{\circ} 3$, p. 9-31, https://www.erudit.org/fr/revues/ circuit/2004-v14-n3-circuit3618/902324ar/, consulté le 28 août 2017.

Becker, Howard S. ([1982]1988), Les mondes de l'art, traduit de l'anglais par Jeanne Bouniort, présentation de Pierre-Michel Menger, Paris, Flammarion.

Berio, Luciano (1971), « Luciano Berio. Commentaires au rock », Musique en jeu, n 2, p. 56-65.

Bilodeau, Julien (2017), Another Brick in the Wall. Opéra en deux actes. D'après les paroles et la musique de The Wall de Rogers Waters, partition non publiée et transmise à l'auteur du présent article en version PDF.

Bourdieu, Pierre (1979), La distinction. Critique sociale du jugement, Paris, Éditions de Minuit.

Cormier, Sylvain (2017), "Another Brick in the Wall - l'opéra. Frapper un mur ", Le Devoir, http://www.ledevoir.com/culture/musique/493790/another-brick-in-the-wall-1-opera-frapper-unmur, consulté le 20 mars 2017.

Dufour, Pierre (2017), "La genèse du projet », Another Brick in the Wall-l'opéra. Programme, page non identifiée.

Durocher, Sophie (2017), "The Wall sans The Wall, c'est pas The Wall », Le Journal de Montréal, http://www.journaldemontreal.com/2017/03/13/emthe-wall-em--sans-emthe-wall-em-cest-pasemthe-wall-em, consulté le 20 mars 2017. 
Farber, Jim (2017), « Pink Floyd's The Wall Finds Scary Modern relevance as New Opera », Observer, http://observer.com/2017/03/pink-floyd-another-brick-in-the-wall-opera-review/, consulté le 20 mars 2017.

Fryer, Paul (2014), Opera in the Media Age. Essays on Art, Technology and Popular Culture, Jefferson, McFarland \& Company Inc.

Goldman, Jonathan (2014), "Introduction sous le signe de la guitare électrique », Circuit, musiques contemporaines, vol. 24, $\mathrm{n}^{\circ} 3$, p. 5-8, https://www.erudit.org/fr/revues/circuit/2014-v24-n3circuit01610/, consulté le 28 août 2017.

Gonneville, Michel (2012), "Entendu dans Cette ville étrange. L'un de nous ", Circuit, musiques contemporaines, vol. 22, $\mathrm{n}^{\circ} 1, \mathrm{p} .103-108$.

Grow, Kory (2017), " Pink Floyd's The Wall Opera Trades Rock for Emotional Power », Rolling Stone, http://www.rollingstone.com/music/live-reviews/review-pink-floyds-new-the-wallopera-w471698, consulté le 20 mars 2017.

Hennion, Antoine (1993), La Passion musicale. Une sociologie de la médiation, Paris, Métailié.

Huss, Christophe (2017), "Another Brick in the Wall - l'opéra. Le second acte est le bon ! ", Le Devoir, http://www.ledevoir.com/culture/musique/493789/another-brick-in-the-wall-1-opera-le-secondacte-est-le-bon, consulté le 20 mars 2017.

Houle, Nicolas (2017), "The Wall. L'opéra rock devenu opéra », Le Soleil, http://www.lapresse.ca/ le-soleil/arts/sur-scene/201703/11/01-5077925-the-wall-lopera-rock-devenu-opera.php, consulté le 20 mars 2017.

Jauss, Hans Robert ([1972]1978), Pour une esthétique de la réception, traduit de l'allemand par Claude Maillard, préface de Jean Starobinski, Paris, Gallimard.

Jones, Carys Wyn (2008), The Rock Canon. Canonical Values in the Reception of Rock Albums, Burlington, Ashgate.

Kelly, Brendan (2017), "Brendan Kelly. Mixed Reviews Unlikely to Dampen Enthusiasm for Another Brick in the Wall - The Opera », Montreal Gazette, http://montrealgazette.com/entertainment/ brendan-kelly-mixed-reviews-unlikely-to-dampen-enthusiasm-for-another-brick-in-the-wall-theopera, consulté le 20 mars 2017.

Lacombe, Hervé (2007), Géographie de l'opéra au XXe siècle, Paris, Fayard.

Leroy, Aymeric (2009), Pink Floyd. Plongée dans l'œuvre d'un groupe paradoxal, Marseille, Le mot et le reste.

Lipscomb, Scott, et Joe Stuessy ([2001]2013), Rock and Roll. Its History and Stylistic Development, $7^{\mathrm{T}}$ éd., Boston, Pearson.

Machart, Renaud (2004), John Adams, Arles, Actes Sud.

McKinley, Maxime (2014), "Trajectoire d'une connexion. Romitelli/Montréal ", Circuit, musiques contemporaines, vol. 24, no 3, p. 69-77, https://www.erudit.org/fr/revues/circuit/2014-v24-n3circuit01610/1027611ar/, consulté le 28 août 2017.

Pedler, Emmanuel (2004), « Entendement musical et malentendu culturel. Le concert comme lieu de confrontation symbolique ", Sociologies et Sociétés, vol. xxxvi, n 1, p. 127-144.

Pirenne, Christophe (2011), Une histoire musicale du rock, Paris, Fayard.

Ramaut-Chevassus, Béatrice (2003), "Opéra et postmodernité. L'exemple de John Adams »,dans Béatrice Ramaut-Chevassus (dir.), Composer un opéra aujourd'hui, Actes de la journée d'étude du 13 mai 2003, Saint-Étienne, Publications de l'Université de Saint-Étienne, p. 107-124.

Repentigny, Alain de (2017), "Another Brick in the Wall. Une relecture utile ", La Presse, http://www.lapresse.ca/arts/spectacles-et-theatre/critiques-de-spectacles/201703/13/015078191-another-brick-in-the-wall-une-relecture-utile.php, consulté le 20 mars 2017.

Rodgers, Caroline (2017), "Another Brick in the Wall. Spectaculaire et déroutant ", La Presse, http://www.lapresse.ca/arts/spectacles-et-theatre/critiques-de-spectacles/201703/13/015078192-another-brick-in-the-wall-spectaculaire-et-deroutant.php, consulté le 20 mars 2017. 
Salzman, Eric, et Thomas Desi (2008), The New Music Theater. Seeing the Voice, Hearing the Body, Oxford, Oxford University Press.

Shute, Gareth (2013), Concept Albums, UsA, Investigations Publishing.

Taruskin, Richard (2005), The Oxford History of Western Music, « Vol. 5. The Late Twentieth Century », Oxford, Oxford University Press.

Tremblay, Odile (2017), "Assourdir le rock », Le Devoir, http:/ /www.ledevoir.com/culture/actualitesculturelles/494028/chronique-odile-tremblay, consulté le 20 mars 2017.

Trottier, Danick (2017), « Les compositeurs face au passé musical. L'attrait pour l'écriture orchestrale dans la trajectoire de quelques compositeurs québécois", Circuit, musiques contemporaines, vol. 27, $\mathrm{n}^{\mathrm{o}} 1$, p. 25-39.

Wicke, Peter ([1987]1995), Rock Music. Culture, Aesthetics and Sociology, traduit par Rachel Fogg, Cambridge (UK), Cambridge University Press. 